ORIGINAL ARTICLE

\title{
The relationship between job satisfaction and health: a meta-analysis
}

\author{
E B Faragher, M Cass, C L Cooper
}

Occup Environ Med 2005;62:105-112. doi: 10.1136/oem.2002.006734

Background: A vast number of published studies have suggested a link between job satisfaction levels and health. The sizes of the relationships reported vary widely. Narrative overviews of this relationship have been published, but no systematic meta-analysis review has been conducted.

Methods: A systematic review and meta-analysis of 485 studies with a combined sample size of 267995 individuals was conducted, evaluating the research evidence linking self-report measures of job satisfaction to measures of physical and mental wellbeing.

See end of article for authors' affiliations

Results: The overall correlation combined across all health measures was $r=0.312(0.370$ after SchmidtHunter adjustment). Job satisfaction was most strongly associated with mental/psychological problems; strongest relationships were found for burnout (corrected $r=0.478$ ), self-esteem $(r=0.429$ ), depression

Correspondence to: Dr E B Faragher,

Manchester Business

School, The University of

Manchester, Booth Street

West, Manchester M15

6PB, UK; brian.faragher@

manchester.ac.uk $(r=0.428)$, and anxiety $(r=0.420)$. The correlation with subjective physical illness was more modest $(r=0.287)$.

Conclusions: Correlations in excess of 0.3 are rare in this context. The relationships found suggest that job satisfaction level is an important factor influencing the health of workers. Organisations should include the development of stress management policies to identify and eradicate work practices that cause most job dissatisfaction as part of any exercise aimed at improving employee health. Occupational health clinicians should consider counselling employees diagnosed as having psychological problems to critically evaluate

Accepted

8 September 2003 their work - and help them to explore ways of gaining greater satisfaction from this important aspect of their life.

$\mathrm{E}$ pidemiologists have long been aware that social and environmental factors can contribute to the incidence of many human diseases. Predictably, as the single activity occupying most people's waking time is work, pressures, strains, and stresses within the workplace have been identified as being a potentially important health factor. Numerous theories now exist, developed from a wide range of perspectives, postulating a direct link between organisational/workplace stress and wellbeing. ${ }^{1}$

There is growing evidence that current trends in employment conditions may be eroding levels of job satisfactionand directly damaging the physical and mental health of employees. ${ }^{2}$ New working practices and rapid technological advances are changing the nature of many jobs. ${ }^{3}$ Employees are regularly being required to work well beyond their contracted hours, often unwillingly, as organisations struggle to meet tight deadlines and targets. ${ }^{4}$ Work practices are becoming more automated and inflexible, leaving employees with less and less control over their workload. Many organisations are reducing their permanent workforce and converting to a culture of short term contracts or "out-sourcing", increasing feelings of job insecurity. These trends have undoubtedly contributed considerably to the development of a "workaholic" culture throughout the UK and Europe-a climate which is impacting negatively on the levels of enjoyment and satisfaction employees gain from their work.

Governments in many developed industrial countries have already drafted —or are drafting—-legislation to make organisations accept greater responsibility for the impact of their work practices on the health of their employees. Consequently, an increasing number of employers are developing formal stress intervention/management policies. Ideally, such policies should be evidence based. However, in the past two decades alone, considerable research effort has been directed at issues relating to work related stress and health. The research database is already enormous - and is growing at a considerable rate. Any organisation starting to review the available evidence is likely to be overwhelmed by the enormity of the task. Many different workplace characteristics have been found to correlate significantly with both stress levels and health-and equally many organisational and environmental factors have been found that potentially moderate the effects of stressors. ${ }^{5}$ Furthermore, if reviewed superficially, the evidence can easily appear to be both contradictory and inconclusive.

Clearly, there is an urgent need for a systematic and thorough review of the research evidence linking work related stress factors with ill health. The HERMES (Health and Employment Review: a MEta-Analysis Study) project was instigated to start this task, funded initially by a grant from the UK Health and Safety Executive. Of the factors evaluated so far within this project, employee self-reported job satisfaction has emerged as having by far the strongest link with employee wellbeing. The findings of this part of the HERMES project are reported below.

Previous meta-analyses have reported important relationships between important life/work characteristics and job satisfaction, most notably life satisfaction ${ }^{6}$ and job performance. ${ }^{7}$ Using exactly the same statistical methodology-but with a more comprehensive review of the literature using electronic methods not available to earlier authors and an extensive search for "grey literature" and unpublished reports-this paper reports the finding of an exhaustive systematic review/ meta-analysis of the available research examining the relationship between job satisfaction and both physical and mental health.

\section{METHODS}

Rationale for the use of meta-analysis

As already stated, there are many, often apparently contradictory, published studies linking job satisfaction to measures 
of health. Standard narrative literature review methods are inadequate in this situation; more quantitative methods are needed. The statistical methods of meta-analysis were developed to meet this need. These enable the quantitative results of several individual studies to be amalgamated and provide a combined estimate of effect size (that is, in this context, of the correlation between job satisfaction and health). Meta-analytical statistics are based on much larger sample sizes and yield weighted average effect size estimates that are more accurate than those from individual studiesand are sensitive to findings of different magnitude across studies. ${ }^{8}$ Most importantly, meta-analysis methods enable researchers to determine the strength and direction of relationships after eliminating variance due to statistical artefacts such as sampling and measurement error. ${ }^{6}$

\section{Measures \\ Job satisfaction}

Some theorists view job satisfaction as being the positive emotional reactions and attitudes an individual has towards their job." Others have viewed it as a bi-dimensional construct consisting of "intrinsic" and "extrinsic" satisfaction dimensions, ${ }^{10}$ or alternatively of "satisfaction/lack of satisfaction" and "dissatisfaction/lack of dissatisfaction" dimensions. ${ }^{11}$ More recently, debate has arisen as to whether job satisfaction is a global concept or is composed of facets of satisfaction with various aspects of an individual's job. ${ }^{92} \mathrm{~A}$ recent study ${ }^{13}$ has suggested that the most important determinants of job satisfaction are whether an employee finds their job interesting, has good relationships with their managers and colleagues, has a high income, is allowed to work independently, and has clearly defined career advancement opportunities.

Measures of job satisfaction tend to fall into two broad types: single item global measures and composite measures of satisfaction with various job components. Those most commonly found in this review were the Warr Job Satisfaction Questionnaire, ${ }^{14}$ the Occupational Stress Indicator, ${ }^{15}$ the Michigan Organisational Assessment Questionnaire ${ }^{16}$ the Job Diagnostic Survey, ${ }^{17}$ the Job Descriptive Index "work itself" subscale, ${ }^{18}$ the Minnesota Satisfaction Questionnaire, ${ }^{19}$ and the Brayfield-Rothe Questionnaire. ${ }^{20}$ All are self-report, multi-item questionnaires.

\section{Health outcome}

Health outcome measures were divided into those evaluating mental health and those relating to physical health. Mental health scales measuring depression, anxiety, burnout, selfesteem, and general mental health (predominantly hybrid scales measuring elements of depression and anxiety) were accepted; physical health scales were restricted to subjective scales (mostly measuring a combination of "psychosomatic complaints" such as headaches, dizziness, muscle pain, and digestive problems), cardiovascular disease, and musculoskeletal disorders. The condition "strain" was created to allow studies which combined mental and physical health measures to be included.

Most self-rated health scales measure ill health, with high scores denoting high levels of physical or mental disorder. High levels of job satisfaction were hypothesised as being associated with improved health. All correlations were converted to reflect the extent to which they confirmed (or refuted) this hypothesis (for example, if job satisfaction correlated negatively with a measure of $i l l$ health, the correlation was evaluated as a positive correlation between job satisfaction and good health).

\section{Procedures}

The procedures used to complete the systematic review and meta-analysis were based on established best practice, ${ }^{821}$ and incorporated the relevant elements of the QUORUM statement. The Cochrane Collaboration (http://www.cochrane. org) was created in 1992 with the purpose of "preparing, maintaining, and promoting the accessibility of systematic reviews of the effects of healthcare interventions". Of its many major contributions to the field of healthcare research, one of its most important has been the creation of guidelines for the execution and reporting of meta-analyses and systematic reviews. While these were prepared primarily for reviews of randomised controlled trials, many of the principles enshrined in the guidelines hold also for the combination of the results of observational/correlational studies.

\section{Formulation of the review question}

The study objective was to systematically review the research evidence linking job satisfaction to measures of health, and to subject this evidence to a series of meta-analyses to obtain combined estimates of the strengths of these statistical relationships. The literature contains a number of studies giving a narrative review of these relationships but, to our knowledge, no systematic meta-analysis review has been published.

\section{Inclusion/exclusion criteria}

Intervention and observational studies were accepted if:

- An evaluation of the relationship between a measure of job satisfaction and a relevant health measure was reported, using one of the following effect-size statistics: correlation coefficient, linear regression coefficient (preferably standardised), $\mathrm{R}^{2}$ values from (multiple) regression analyses, odds-ratios/prevalence ratios, significant test statistic (Student's $t, \mathrm{~F}$ ratio, or $\chi^{2}$ ), group means (or mean differences)

- A normal working population was studied

- Acceptably large samples (at least 30 respondents in total) were evaluated

- One of the following prospective or retrospective experimental designs was used: cross-sectional/correlational cohort, longitudinal cohort, case-control comparison, randomised control trial, group differences based on existing criteria

- The study report was published after 1970.

\section{Search for and location of eligible studies}

An initial comprehensive electronic search for relevant literature was carried out using the databases PsychInfo, PubMed, Social Sciences Citation Index, Arts \& Humanities Citation Index, and ERIC. A list of all journals that might potentially yield eligible studies (irrespective of publication language) was then compiled and confirmation obtained that all were included in the databases searched. Any remaining journals were scanned using their individual websites or other (non-electronic) databases.

Stress Medicine, Work \& Stress, and the Journal of Occupational and Organisational Psychology were then hand searched for relevant articles published in the past five years. For every paper found that had been missed by the electronic search, the reasons were determined, a revised list of keywords created, and the electronic searches repeated.

As not all research studies are published in mainstream journals, the so-called "grey literature" was then searched, including conference abstracts, thesis/dissertation abstracts, non-refereed journals, government reports, and technical reports. Finally, leading academics known to be actively researching in this field were written to with a request for details about any relevant unpublished studies owned by 
them and for contact addresses for other researchers known to them who might have unpublished studies.

\section{Establishment of methodological criteria}

Less methodologically rigorous studies are more likely to produce inflated effect sizes (that is, to overestimate the strengths of the relationships between job satisfaction and health). Well established and agreed measures of methodological rigour have been established by the Cochrane Collaboration for combining randomised controlled trials in a meta-analysis, but a thorough search failed to identify criteria suitable for correlational studies. A measure of methodological rigour was thus developed specifically for this meta-analysis.

The literature was scanned for guidelines on research procedures in organisational psychology. The methodological criteria identified were added to those used for medical studies, to create a list of 26 criteria potentially relevant for assessing methodological quality in correlational studies. Six experienced researchers in the field of organisational psychology were asked to examine these criteria and to rank in order the 10 they considered most important for organisational psychology research. The following 10 criteria were rated clearly higher than all others:

- Sample representative of organisation(s) studied

- Sample stratified for gender, age, level of seniority, ethnic origin, or degree level

- Summary statistics for gender, degree level, occupation, industry, country, age

- Response rate adequate

- Sample size acceptable

- Job satisfaction and health outcome measures acceptable (that is, validated, reliable, internally consistent and either continuous or ordinal)

- Useable effect size statistic(s) reported (or obtainable from authors)

- Statistical analysis of study findings appropriate

- Adjustment made for important confounding factors

- Attrition rate adequate (if longitudinal design used).

Each study considered eligible for the meta-analysis was rated according to these criteria $(0=$ unacceptable; $1=$ acceptable) and a summated "rigour" score computed (range 0$10)$.

\section{Statistical methods}

Because of the nature of the studies being reviewed, the vast majority of reports provided effect sizes in the form of correlation coefficients or regression coefficients. In those instances where other types of effect size statistic were reported (for example, odds ratios, mean differences, etc), these were converted to correlation coefficients using standard formulae. ${ }^{8122}$

For each study that passed the review quality criteria detailed above, a correlation table was then constructed containing the correlations between all of the measures, the reliability alpha coefficients for each measure, and an indicator of whether or not each correlation was in the expected direction. This information was then entered into the "Comprehensive Meta-Analysis (CMA)" computer program. ${ }^{23}$

Initially, the effects of sampling error were corrected for by replacing the distribution of observed correlations with the distribution of population correlations. The mean of the latter distribution was estimated by the mean of the observed correlations weighted by sample size; the variance was estimated by subtracting the variance due to sampling error from the variance of the observed correlations (this estimates the actual variation in the study correlations without the extra (nuisance) variance due to sampling error).

Correlation coefficients tend to be biased towards zero because of artefacts. In most of the studies eligible for inclusion in this review, job satisfaction and health were both usually measured with imperfect statistical reliability (conventionally measured using the Cronbach alpha coefficient). This can attenuate the magnitude of the observed correlations, so the Schmidt and Hunter correction formula ${ }^{8}$ was applied to each correlation to adjust for the reliability of the measures used. Where reported, the actual study reliability statistic was used; otherwise, the published "norm" reliability was used.

A formal significance tests for heterogeneity between the studies included in the meta-analysis was statistically significant, indicating considerable study-to-study variation. However, this test has low power and must be treated with caution. There were no obvious technical or scientific reasons for excluding any of the studies, so all were retained in the analysis. The conclusions drawn are based on the random effects model estimates of the combined correlations.

Two sensitivity analyses were carried out to investigate the influence on the combined correlation estimates of (a) the decade in which the study was published and (b) the rigorousness of the study (as determined by the "rigour" score described above). Although there were many other potential factors that could have increased the variation between the studies evaluated (primarily, range restriction of scales and differing validities), there was insufficient information provided in the vast majority of reports to make the necessary corrections. Similarly, too few studies provided information on the characteristics of the study sample to permit informative sensitivity analyses other than those described above.

\section{RESULTS}

The expected relationship was that an increase in job satisfaction would be associated with improved health. The unadjusted and adjusted combined correlation estimates are summarised in table 1; the corresponding Forrest plots are shown in fig 1 . The effects of both year of study and "rigour" rating are summarised in table 2 .

The overall combined studies relationship found between job satisfaction and (good) health was indeed positive $(\mathrm{r}=0.312$, adjusted $\mathrm{r}(\check{\mathrm{r}})=0.370)$. Job satisfaction was much more strongly associated with mental/psychosocial problems than with physical complaints. The largest relationship was found for burnout $(\mathrm{r}=0.409, \check{\mathrm{r}}=0.478)$; only five of the 62 studies into burnout identified failed to reach statistical significance.

Job satisfaction also correlated positively, but slightly less strongly, with the other mental health characteristics considered: depression $(\mathrm{r}=0.366, \breve{\mathrm{r}}=0.428)$; anxiety $(\mathrm{r}=0.354$, $\check{\mathrm{r}}=0.420)$; self-esteem $(\mathrm{r}=0.351, \breve{\mathrm{r}}=0.429)$; general mental health $(\mathrm{r}=0.318, \check{\mathrm{r}}=0.376)$. While the relationship between job satisfaction and strain was also relatively high $(r=0.310$, $\check{\mathrm{r}}=0.341$ ), the correlation with subjective physical illness was more modest $(\mathrm{r}=0.235, \check{\mathrm{r}}=0.287)$.

The lowest correlations were found for the two physical illnesses studied: cardiovascular disease $(\mathrm{r}=0.113, \check{\mathrm{r}}=0.121)$ and musculoskeletal disorders $(r=0.078, \check{r}=0.079)$.

Studies published since 1990 tended to produce similar or slightly smaller correlations than those completed before that date, with the exception of general mental health (for which the correlation increased slightly). The "rigour" rating given to each study had a slightly stronger but inconsistent influence; highly rated studies involving general mental health tended to produce the highest correlations with job 
Table 1 Effect-size summary statistics for relationship between job satisfaction and health measures

\begin{tabular}{|c|c|c|c|c|c|}
\hline \multirow[b]{2}{*}{ Health outcome } & \multirow{2}{*}{$\begin{array}{l}\text { No. of } \\
\text { studies }\end{array}$} & \multirow{2}{*}{$\begin{array}{l}\text { Total sample } \\
\text { size }\end{array}$} & \multicolumn{2}{|c|}{ Combined correlation coefficient $(95 \% \mathrm{Cl})$} & \multirow[b]{2}{*}{ Heterogeneity test } \\
\hline & & & Fixed effects model & Random effects model & \\
\hline \multicolumn{6}{|l|}{ Unadjusted } \\
\hline Anxiety & 60 & 36443 & $0.322(0.313$ to 0.331$)$ & $0.354(0.319$ to 0.388$)$ & $Q(59)=650.7, p<0.001$ \\
\hline Burnout & 62 & 19944 & $0.396(0.385$ to 0.408$)$ & 0.409 (0.378 to 0.439$)$ & $Q(61)=355.5, p<0.001$ \\
\hline Cardiovascular disease & 13 & 5303 & $0.147(0.120$ to 0.173$)$ & $0.113(0.041$ to 0.183$)$ & $Q(12)=55.0, p<0.001$ \\
\hline Depression & 46 & 38941 & $0.349(0.341$ to 0.358$)$ & $0.366(0.310$ to 0.421$)$ & $Q(45)=1553.1, p<0.001$ \\
\hline General mental health & 142 & 95814 & $0.331(0.326$ to 0.337$)$ & $0.318(0.299$ to 0.336$)$ & $Q(141)=1130.7, p<0.001$ \\
\hline Musculoskeletal disorders & 4 & 2442 & $0.078(0.039$ to 0.118$)$ & $0.078(0.039$ to 0.118$)$ & $Q(3)=2.3, p=0.519$ \\
\hline "Other" illness & 3 & 2124 & $0.315(0.276$ to 0.353$)$ & $0.251(-0.167$ to 0.593$)$ & $Q(2)=124.4, p<0.001$ \\
\hline Subjective physical illness & 119 & 58762 & $0.228(0.220$ to 0.235$)$ & $0.235(0.211$ to 0.259$)$ & $Q(118)=937.8, p<0.001$ \\
\hline Self-esteem & 13 & 2529 & $0.345(0.310$ to 0.379$)$ & 0.351 (0.251 to 0.443$)$ & $Q(12)=80.9, p<0.001$ \\
\hline Strain & 23 & 5693 & $0.319(0.295$ to 0.342$)$ & $0.310(0.230$ to 0.385$)$ & $Q(22)=208.8, p<0.001$ \\
\hline Combined & 485 & 267995 & $0.310(0.306$ to 0.313$)$ & $0.312(0.299$ to 0.325$)$ & $Q(487)=6191.8, p<0.001$ \\
\hline \multicolumn{6}{|l|}{ Schmidt-Hunter adjusted } \\
\hline Anxiety & 60 & 36443 & $0.383(0.374$ to 0.392$)$ & $0.420(0.379$ to 0.459$)$ & $Q(59)=1051.7, p<0.001$ \\
\hline Burnout & 62 & 19944 & $0.463(0.452$ to 0.474$)$ & $0.478(0.443$ to 0.512$)$ & $Q(61)=534.7, p<0.001$ \\
\hline Cardiovascular disease & 13 & 5303 & $0.163(0.136$ to 0.189$)$ & $0.121(0.043$ to 0.197$)$ & $Q(12)=65.4, p<0.001$ \\
\hline Depression & 46 & 38941 & $0.412(0.404$ to 0.421$)$ & $0.428(0.361$ to 0.490$)$ & $Q(45)=2431.1, p<0.001$ \\
\hline General mental health & 141 & 95814 & $0.393(0.388$ to 0.399$)$ & $0.376(0.353$ to 0.397$)$ & $Q(142)=1778.8, p<0.001$ \\
\hline Musculoskeletal disorders & 4 & 2442 & $0.079(0.039$ to 0.118$)$ & $0.079(0.039$ to 0.118$)$ & $Q(3)=2.6, p=0.452$ \\
\hline "Other" illness & 3 & 2124 & $0.360(0.323$ to 0.397$)$ & $0.286(-0.201$ to 0.660$)$ & $Q(2)=170.6, p<0.001$ \\
\hline Subjective physical illness & 119 & 58762 & $0.272(0.265$ to 0.280$)$ & $0.287(0.255$ to 0.319$)$ & $Q(118)=1886.3, p<0.001$ \\
\hline Self-esteem & 13 & 2529 & $0.439(0.407$ to 0.470$)$ & $0.429(0.304$ to 0.540$)$ & $Q(12)=144.2, p<0.001$ \\
\hline Strain & 24 & 5693 & $0.355(0.333$ to 0.377$)$ & $0.341(0.250$ to 0.426$)$ & $Q(22)=320.8, p<0.001$ \\
\hline Combined & 485 & 267995 & $0.367(0.364$ to 0.371$)$ & $0.370(0.354$ to 0.385$)$ & $Q(484)=10028.1, p<0.001$ \\
\hline
\end{tabular}

satisfaction, while correlations tended to decrease with increased rating for physical illness, cardiovascular disease, and strain.

\section{DISCUSSION}

This paper reports a meta-analysis of almost 500 studies of job satisfaction, incorporating over 250000 employees in a large variety of different organisations based throughout the world. The largest combined statistical correlations found were between job satisfaction and measures of mental health; smaller relationships were detected for measures of physical health. All of the correlations were positive and highly statistically significant. The authors of this paper contend that the combined correlations were sufficiently large numerically to be considered as being both strong and extremely important.

The studies accepted for inclusion in the analysis were predominantly cross-sectional and observational. Furthermore, the high levels of statistical significance obtained for the correlation estimates were virtually inevitable given the very large sample size represented by the combined studies data set (the statistical significance level for a correlation coefficient is related directly to the size of the sample from which it is estimated). Thus, a causal relationship between employee health and job satisfaction cannot be automatically inferred directly from the statistical evidence. A consideration of the psychological issues involved is also needed.

In the context of this review, however, causal inferences do appear to be very plausible. The meta-analysis findings indicate that, on average, employees with low levels of job satisfaction are most likely to experience emotional burn-out, to have reduced levels of self-esteem, and to have raised levels of both anxiety and depression. Many people spend a considerable proportion of their waking hours at work. If their work is failing to provide adequate personal satisfaction-or even causing actual dissatisfaction-they are likely to be feeling unhappy or unfulfilled for long periods of each working day. It seems reasonable to hypothesise that such individuals are at increased risk of experiencing a lowering of general mood and feelings of self-worth while at work, culminating in mild levels of depression and/or anxiety. If continued unresolved for any length of time, such emotions could eventually lead to emotional exhaustion, particularly if the individual is unable to prevent their feelings from spilling over into their home/social life.

The numerical sizes of the relationships found between job satisfaction and many of the mental health measures are also noteworthy. Correlations involving measures of workplace stress/health rarely exceed $r=0.333$ (that is, $R^{2}=10 \%$ ). In this meta-analysis, the corrected combined correlations between job satisfaction and each of burnout, self-esteem, depression, anxiety, and general mental health were well in excess of this figure. The importance of such strong correlations should not be underestimated. For example, the corrected combined correlation between job satisfaction and burnout was $\check{r}=0.478$. As the measures were re-scaled for the meta-analysis so that increases in job satisfaction were related to improvements in wellbeing, a one standard deviation decrease in job satisfaction level corresponds statistically to an average increase in symptoms of burnout/ emotional exhaustion of almost one-half of one standard deviation. A modest decrease in job satisfaction levels is therefore associated with an increase in the risk of employee burnout sufficiently large to be of considerable clinical importance.

Interpreting the size of a correlation coefficient has always caused difficulty. Several authors have attempted to provide practical guidelines; the most commonly quoted are those advocated by Cohen. ${ }^{24}$ However, as Rosenthal and colleagues state in their excellent discussion of this issue, "mechanically labelling ... [correlation coefficients] ... automatically as 'small', 'medium' and 'large' can lead to later difficulties ... even 'small' effects can turn out to be practically important". They then cite a number of published examples to prove their case, the most celebrated of which is the major biomedical study $^{25}$ that reported that regular use of aspirin significantly reduced the risk of heart attack in the US population. The findings were so dramatic that the aspirin part of the study was terminated prematurely, with a statistical significance level reported as $\mathrm{p}<0.000001$. Using the data in the study 


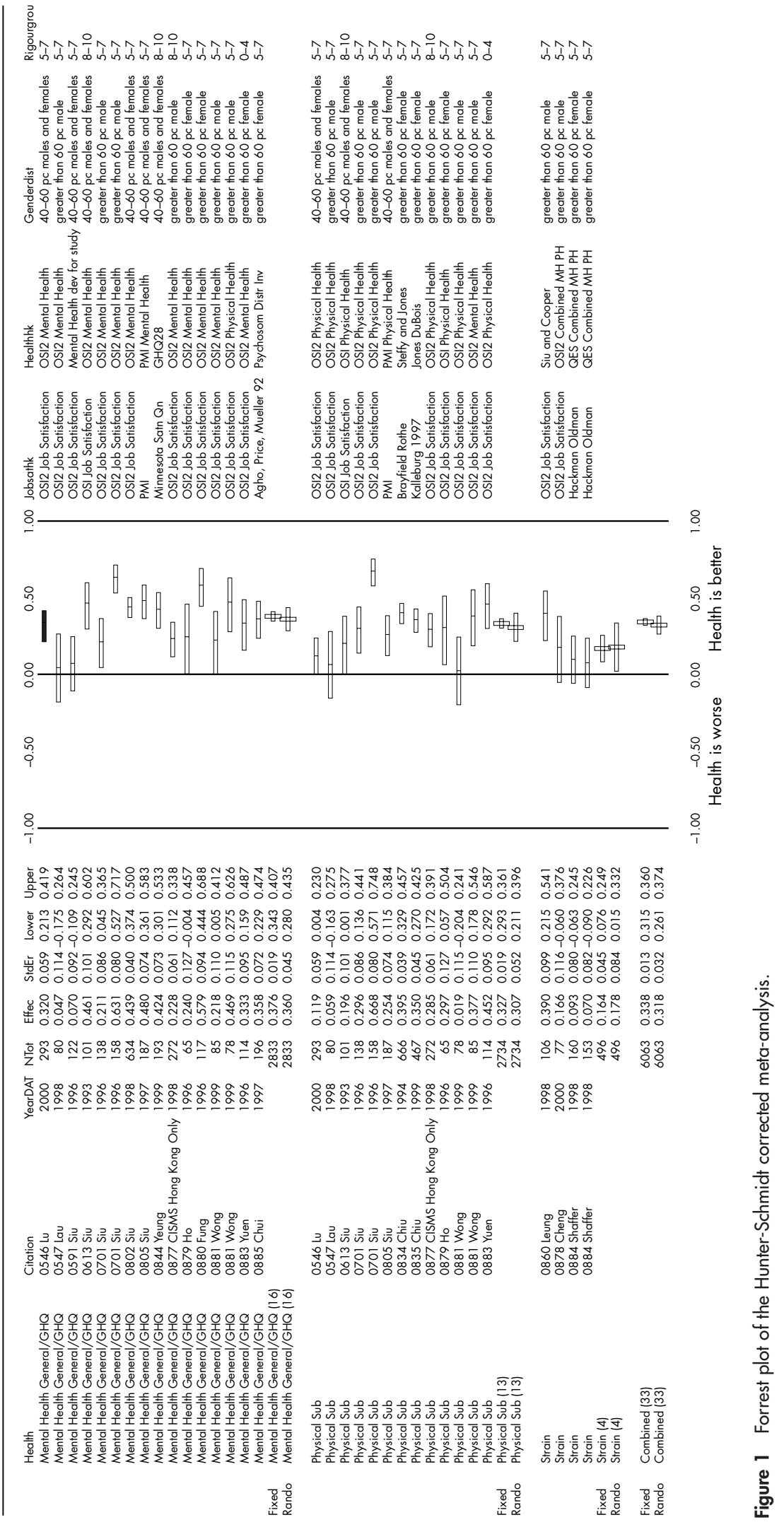




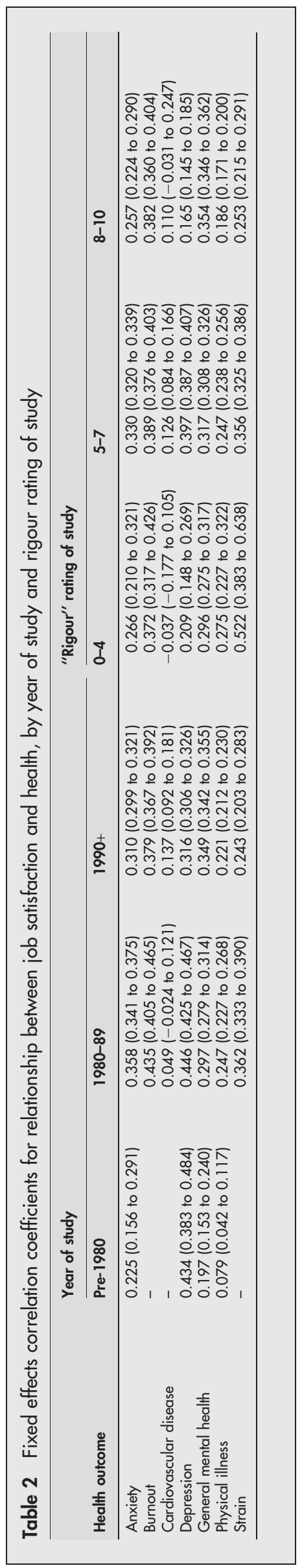

report, the relevant correlation coefficient is very low $(\mathrm{r}=0.034)$. In most other contexts, this would be considered much too small to be of any interest or importance. In fact, this result has saved countless thousands of lives since its publication and has passed into normal clinical practice. The importance of a correlation coefficient is frequently context dependent. We wish to argue that, in the context of observational studies involving primarily "soft"/subjective measures, a combined (adjusted) correlation of 0.370 is, as stated above, "sufficiently large numerically to be considered as being both strong and extremely important".

Equally, the dangers of over-stating the extent of the relationships found must also be avoided. Some researchers ${ }^{26}$ argue cogently that standardised regression coefficients and correlations are imperfect measures of effect-size; unfortunately, the better alternatives suggested are reported for very few studies, and they are not available in the widely used meta-analysis software, so there is no real practical alternative. The vast majority of meta-analyses published combine the results of comparative studies (usually randomised controlled trials), so use odds ratios as the effect-size of choice. However, the studies of interest to this review were almost exclusively observational and so reported correlation/ regression coefficients. While the assumptions underpinning these coefficients can be problematic statistically (for example, linear relationships usually have to be assumed), the techniques for combing these are well developed.

While their average impact on individual employees may be important, even very large coefficients account for only a modest amount of overall variation in health levels. As stated above, the corrected correlation between job satisfaction and burnout was $\check{r}=0.478$, corresponding to an $R^{2}$ value of just $22.8 \%$. That is, less than one-quarter of the variation in burnout scores is accounted for. While job satisfaction may have a clearly discernible impact on this aspect of mental health, there are also many other factors involved (at least $77.2 \%$ of the variation in burnout ratings remain unaccounted for).

In common with other statistical methods, the results obtained from a meta-analysis are subject to various potential sources of bias. A combined effect-size estimate is only as good as the individual values from which it is composedthere are no standards widely adopted for the presentation of observational studies, so the studies included were of very variable quality. Some study reports indicated that measures of both job satisfaction and health were included, but no useable effect-size statistics were reported; this would suggest that there may be a bias in favour of significant correlations, inflating the combined estimate. However, a funnel plot of the effect-sizes included in the meta-analysis suggested that this was not a major problem. Finally, the great majority of studies used self-report measures of both job satisfaction and health, so there will have been some inflation of the individual correlation coefficients due to shared variance.

There are issues also with the concept of job satisfaction itself. Job satisfaction is generally measured by asking individuals to rate individual facets of their work and then aggregating these into a single (global) score. The widely used Job Descriptive Index ${ }^{18}$ measures five facets of job satisfaction, specifically satisfaction with the work itself, pay, promotion, supervision, and co-workers. Working hours, job security, supervisor support, and changes in job control levels have also been related to individual job satisfaction levels. ${ }^{27} 28$ Some critics argue, however, that facet measures of job satisfaction should be separated from overall satisfaction, as they are conceptually different. ${ }^{29}$ Overall satisfaction is essentially the global attitude or feeling an individual has about their job as a whole and may be related to internal 
characteristics such as personality. Satisfaction levels can vary markedly between different job facets-and these in turn may be different from an employee's overall feeling of job satisfaction. Theoretically, an individual may have high satisfaction with many facets of their job but still feel overall job dissatisfaction (for example, they may enjoy the facets of their job but be unhappy about the organisation they are working in). Within the context of this study, however, such a distinction may be largely academic: individuals with low levels of satisfaction either with facets of their job or with their job overall may be at increased risk of experiencing an adverse effect on their mental health.

The papers accepted into this review mostly reported studies carried out in Western Europe, the USA, and Australasia, but many studies were included from other, very different, geographical areas (for example, Asia). This raises a problem of combining studies from different cultures, where attitudes to and perceptions of job satisfaction may differ considerably, and from a wide variety of occupations. Certainly, measurement of very subjective concepts such as job satisfaction should be tailored to deal with important cultural and occupational differences-although most studies tended to use one of a small number of standard and validated scales. However, the authors are unconvinced that the relationship between job satisfaction and health will be affected greatly by either cultural or occupational factorsand no evidence of this was found. Lack of work satisfaction is likely to have a negative impact on an individual's feelings about themselves and their life, leading to a reduction in health (particularly mental health), irrespective of type of work and culture.

Despite the reservations expressed above-and even allowing for some bias leading to an inflated estimate of the overall effect size/correlation-the emergence of job satisfaction as by far the highest statistical correlate with health of the workplace characteristics systematically reviewed so far has important implications. Organisations are being required to accept greater responsibilities for the impact of their work practices on employee health levels. Recent research suggests that effective stress intervention policies require good communications between management and employees. ${ }^{30}{ }^{31}$ Workplace policies aimed at improving employee health should be developed through a meaningful dialogue between employees and managers to identify those facets of current work practices that are causing most stress. This meta-analysis suggests that any changes implemented should be monitored for their impact on job satisfaction as well as on employee stress levels. Indeed, there may be a case for identifying those aspects of work causing most job dissatisfaction, and then implementing agreed changes aimed at improving job satisfaction levels. Although organisations may find it easier to influence facet elements of job satisfaction, ways of improving global satisfaction (for example, by changing management practices) need to be explored. Importantly, changes implemented must be desired by the employees and may need to be flexible. For example, increasing job control levels may help some employees-but might actually result in decreased job satisfaction in those who prefer to have their work schedules rigidly defined.

Organisations should not assume that changes made to work practices will be automatically effective. Rigorous evaluation is needed using relevant organisational and employee measures, with the latter including health factors such as sickness and absenteeism rates. However, changes in health levels may take some time (even years) to manifest, whereas improvements in facet and/or global job satisfaction levels may be more immediately discernible. A good job satisfaction scale, developed for the specific needs of the organisation if necessary, must be included as a measure of the effects of the changes made to work practices. If the hypothesised causal link does exist, changes in this scale should predict later changes in overall employee health levels.

Finally, there are equally challenging implications for occupational health professionals. The wellbeing of employees-and in particular their mental health-may be compromised if their work is causing them to experience high levels of dissatisfaction. Thus, the extent to which individuals feel satisfied with their work becomes an important (mental) health issue. In addition to conventional therapeutic approaches, employees diagnosed as having even a mild mental health problem might usefully be counselled to take a hard and critical look at how they feel about their work. If specific tasks they are required to do as part of their job are identified as causing particular dissatisfaction, the employee should be assisted to appropriately change these (for example, by discussing the issue with a manager or colleagues). If the root of the dissatisfaction is more global, a change of job or occupation may be warranted; however, this constitutes a major and stressful life change in itself, so should be considered very carefully.

The notion that health levels can be improved by making changes to work practices intended primarily to enhance facet and/or overall job satisfaction is compelling. Well designed comparative longitudinal studies are now needed in which the effects of workplace interventions on individual employee job satisfaction and health levels are properly monitored. In the meantime, there are good theoretical reasons for hypothesising that job satisfaction is causal linked to health-and particularly to mental wellbeing. Organisations are urged to consider developing stress management policies aimed at identifying and eradicating work practices that cause most job dissatisfaction.

\section{Conclusions}

This large scale meta-analysis of almost 500 studies has provided, for the first time, a clear indication of the immensely strong relationship between job satisfaction and both mental and physical health. The correlations identified are numerically large and highly significant (in both the statistical and clinical sense of the word). The relationships are particularly impressive for aspects of mental health, specifically burnout, lowered self-esteem, anxiety, and depression, where it can now be confirmed that dissatisfaction at work can be hazardous to an employee's mental health and wellbeing. Importantly, the relationships found were much greater than with any other work characteristic evaluated.

This has important health implications for the design and delivery of employee health intervention programmes. The results of this study allow us to conclude that risk assessments of stress in the workplace should attempt to pinpoint those aspects of work that are causing most dissatisfaction among employees (for example, hours of work, organisational management style, workload, work control/autonomy, etc) as these are likely to be also the factors causing raised levels of stress. After meaningful consultation with employees, work practices should be changed appropriately-and the impact of these measured both in terms of their effect on stress levels and on job satisfaction. If the causal relationship hypothesised holds, changes which have the greatest impact on job satisfaction can be expected to produce the greatest benefits to employee mental health (in particular, to reduce levels of burnout/emotional exhaustion)—with a beneficial knock-on effect for organisational health. Occupational health clinicians should also consider counselling employees diagnosed as having a stress related health problem to critically evaluate their work and to explore ways of gaining 
greater satisfaction from this important and time consuming aspect of their life.

\section{ACKNOWLEDGEMENTS}

We thank all members of staff in the School of Management at UMIST who helped in any way with the considerable task of collecting together the studies summarised in this report.

\section{Authors' affiliations}

E B Faragher, M Cass, C L Cooper, BUPA Organisational Psychology and Health Research Group, Manchester School of Management, University of Manchester University of Science and Technology (UMIST), UK

This project was funded by a grant from the UK Health \& Safety Executive

\section{REFERENCES}

1 Cooper CL, ed. The theories of organisational stress. Oxford: Oxford University Press, 1999

2 Kenny DT, Carlson JG, McGuigan FJ, et al, eds. Stress and health: research and clinical applications. Amsterdam: Harwood Academic Publishers, 2000.

3 Cooper CL. Can we live with the changing nature of work? Journal of Managerial Psychology 1999; 14:569-72.

4 Paoli P. First European Survey on the Work Environment 1991-1992. Dublin: European Foundation for the Improvement of Living and Working Conditions, 1992.

5 Cooper CL, ed. Managerial, occupational and organisational stress research. Dartmouth, 2001.

6 Tait M, Padgett MY, Baldwin TT. Job and life satisfaction: a re-evaluation of the strengths of the relationship and gender effects as a function of the date of the study. J Appl Psychol 1989;74:502-7.

7 laffaldno MT, Muchinsky PM. Job satisfaction and job performance: a metaanalysis. Psychol Bull 1985;97:251-73.

8 Hunter JE, Schmidt FL. Methods of meta-analysis: correcting error and bias in research findings. London: Sage, 1990

9 Oshagbemi T. Overall job satisfaction: how good are single vs. multiple-item measures? Journal of Managerial Psychology 1999; 14:388-403.

10 Warr P, Cook J, Wall T. Scales for the measurement of some work attitudes and aspects of psychological well-being. J Occup Psychol 1979;52:129-48.

11 Winefield AH, Tiggemann M, Goldney RD. Psychological concomitants of satisfactory employment and unemployment in young people. Soc Psychiatry Psychiatr Epidemiol 1988;23:149-57.
12 Wanous JP, Reichers AE, Hudy MJ. Overall job satisfaction: how good are single item measures? J Appl Psychol 1997;82:247-52.

13 Sousa-Poza A, Sousa-Poza AA. Well-being at work: a cross-sectional study of the levels and determinants of job satisfaction. Journal of Socio-Economics 2000;29:517-38.

14 Warr P. The measurement of well-being and other aspects of mental health. J Occup Psychol 1990;63:193-210.

15 Cooper CL, Sloan SJ, Williams S. Occupational stress indicator: management guide. Windsor: NFER-Nelson, 1988.

16 Cammann C, Fichman M, Jenkins D, et al. The Michigan Organizational Assessment Questionnaire. University of Michigan, Ann Arbor, 1979.

17 Hackman JR, Oldham GR. Development of the Job Diagnostic Survey. J Appl Psychol 1975;60:159-70.

18 Kinicki AJ, McKee-Ryan FM, Schriesheim CA, et al. Assessing the construct validity of the Job Descriptive Index: a review and meta-analysis. J Appl Psychol 2002;87:14-32.

19 Weiss DJ, Dawis RV, England GW. Manual for the Minnesota Satisfaction Questionnaire. Minnesota Studies in Vocational Rehabilitation (No. 22). 1967.

20 Brayfield AH, Rothe HF. An index of job satisfaction. J Appl Psychol 1951;35:307-11.

21 Lipsey MW, Wilson DB. Practical meta-analysis. Thousand Oaks, CA: Sage, 2001.

22 Rosenthal R, Rosnow RL, Rubin DB. Contrasts and effect sizes in behavioural research. Cambridge: Cambridge University Press, 2000.

23 Borenstein M, Rothstein H, Cohen J. "Comprehensive meta-analysis" manual. New Jersey: Biostat Inc., 2001.

24 Cohen J. Statistical power analysis for the behavioural sciences, rev. edn. New York: Academic Press, 1977.

25 Steering Committee of the Physicians' Health Study Research Group. Preliminary report: findings of the aspirin component of the ongoing physicians' health study. N Engl J Med 1988;318:262-4.

26 Greenland S, Schlesselman JJ, Criqui MH. The fallacy of employing standardised regression coefficients and correlations as measures of effect. Am J Epidemiol 1986; 123:203-8.

27 Burke RJ. Work stressors among recent business school graduates. Stress Med 1998;14:83-9.

28 Noblet A, Rodwell J, McWilliams J. The job strain model is enough for managers: no augmentation needed. Journal of Managerial Psychology $2001 ; 16: 635-49$

29 Scarpello V, Campbell JP. Job satisfaction and the fit between individual needs and organisational rewards. J Occup Psychol 1983;56:315-28.

30 Giga S, Faragher EB, Cooper CL. Identification of good practice in stress prevention management: a state of the art review. Report commissioned by the Health and Safety Executive. University of Manchester Institute of Science and Technology, 2002.

31 Murphy LR, Cooper CL, eds. Healthy and productive work. London: Taylor and Francis, 2000 